\title{
Numerical modeling of space umbrella-type mesh reflector
}

\author{
Sergey Belov 1,*, Aleksey Belkov ${ }^{1}$, Andrey Zhukov ${ }^{1}$, Mikhail Pavlov $^{1}$, Stanislav Kuznetsov ${ }^{1}$ \\ and Sergey Ponomarev ${ }^{1}$ \\ ${ }^{1}$ National Research Tomsk State University, 63450 Tomsk, Russian Federation
}

\begin{abstract}
This paper describes numerical modeling of space umbrellatype mesh reflector. The modeling includes two stages. The first stage embraces the calculation of the shape of cable elements for reflector frontal (rear) nets by the nonlinear force density method. The second stage involves the design of the reflector finite-element model based on the calculated nets. After this, the node displacement and prestress boundary conditions are imposed to determine a node displacement field.
\end{abstract}

\section{Introduction}

Mesh reflectors (MR) are widely used in the space industry due to their relatively small weight and possible large-area deployment. Currently, there exist numerous articles and research investigations devoted to MR modeling [1]. Nevertheless, numerical methods for such structures continue to be challenging. The fact is that such structures include geometrical nonlinear behavior, which, in its turn, requires iterative calculation approaches. In this case, the Newton-Raphson method was applied [2]. Nevertheless, a convergence problem for required solution could arise within this method because of unsatisfactory initial estimation [3].

To calculate the initial reflector equilibrium shape, the nonlinear force density method (FDM) was applied [4] (the first stage of modeling). This method was used in the construction-design of Munich Olympic Stadium [5], the form-finding of space MR cable systems [3], [6] and other structures.

Nonlinear FDM effectively determines the node coordinates of cable elements in an equilibrium state with specific requirements for cable elements (uniform tension, specific distances, etc.). The requirements on the reactions for cable fixed nodes could also be considered [7].

Mathematically, FDM requirements for the cable uniform tensions are described by the nonlinear equations which depend on the force densities of cable elements (constant tension-length ratio). These equations are calculated by applying Newton's iterative method. As a result of linearization, the indefinite system of linear equations was obtained. The solution of linear equations determines the iterative step of force density. Due to infinite number of linear equations solutions, Lagrangian multiplier method was applied [4], [5]. As will be discussed below, ill-conditioned matrix problem became visible in the

*Corresponding author: belovsv@ niipmm.tsu.ru 
process of the reflector frontal (rear) nets form-finding. Ill-conditioned matrix was the result of Jacobian matrices product, whereas Jacobian matrix was a consequence of linearization of nonlinear equations.

To avoid such possible problems with the inversion of ill-conditioned matrix, MoorePenrose pseudoinverse was used [8].

Based on the calculated nets, the reflector finite-element model (FEM) was constructed using link, beam and shell elements (the second stage of modeling).

\section{Stage 1: Calculation of cable node coordinates for reflector networks}

Figure 1 illustrates the reflector model consisting of symmetrical frontal (rear) networks, six-spoke framework and reflecting surface (mesh).
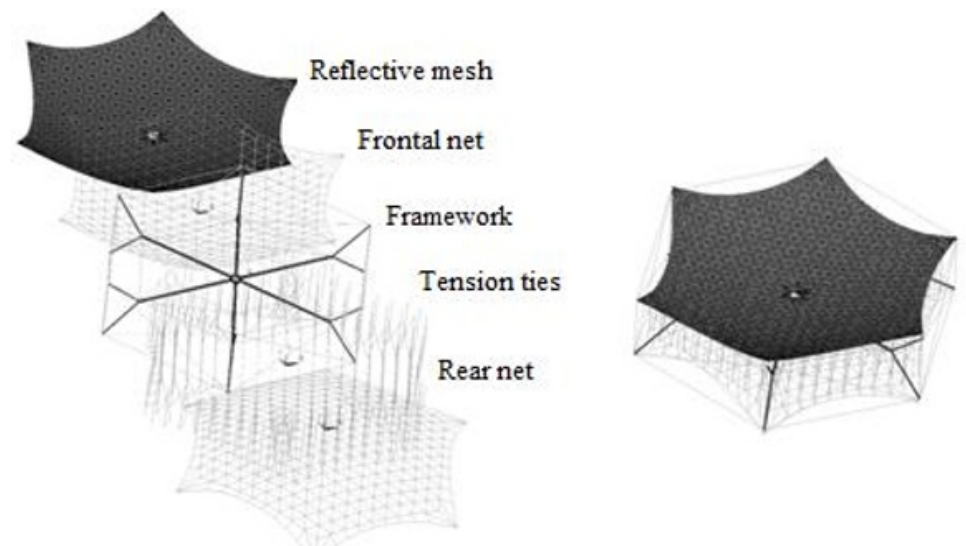

Fig. 1. Reflector model

The framework spoke members are illustrated in figure 2:
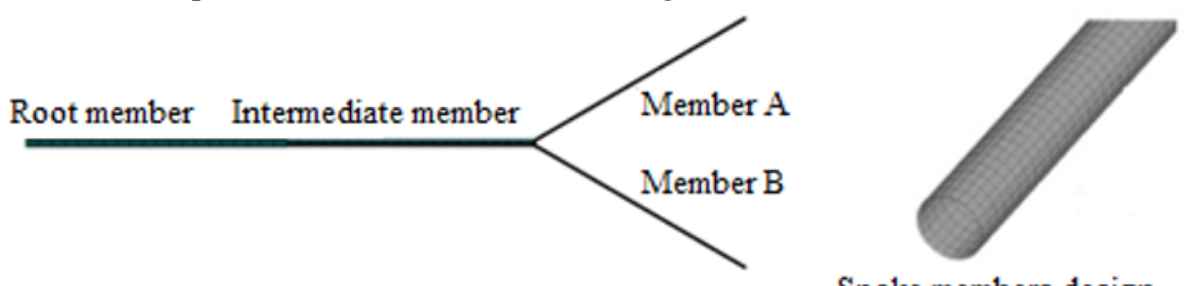

Spoke members design

Fig. 2. Reflector spoke members

To calculate the node coordinates of frontal (rear) net cable elements, a system of equilibrium equations and corresponding nonlinear equations were applied [4]:

$$
\left\{\begin{array}{l}
\left(\mathbf{C}^{T} \mathbf{Q} C\right) \mathbf{x}+\left(\mathbf{C}^{T} \mathbf{Q} \mathbf{C}_{f}\right) \mathbf{x}_{f}=\mathbf{f}_{x} ; \\
\left(\mathbf{C}^{T} \mathbf{Q} C\right) \mathbf{y}+\left(\mathbf{C}^{T} \mathbf{Q} \mathbf{C}_{f}\right) \mathbf{y}_{f}=\mathbf{f}_{y} ; \\
\left(\mathbf{C}^{T} \mathbf{Q C}\right) \mathbf{z}_{\mathrm{par}}+\left(\mathbf{C}^{T} \mathbf{Q} \mathbf{C}_{f}\right) \mathbf{z}_{f}=\mathbf{f}_{z} ; \\
\overline{\mathbf{L}} \overline{\mathbf{q}}-\mathbf{T}_{0}=\mathbf{0} .
\end{array}\right.
$$


where, $\mathbf{C}^{T}[\mathrm{~m} \times \mathrm{n}], \mathbf{C}_{f}\left[m \times n_{f}\right]-$ connectivity matrices for $m$ elements; $n, n_{f}$ - number of unknown and fixed (known) nodes respectively; $\mathbf{Q}[m \times m]=\operatorname{diag}(\mathbf{q})$ - matrix of force densities $q_{j}=T_{j} l_{j} ; \mathbf{x}[n \times 1], \mathbf{y}[n \times 1], \mathbf{x}_{f}\left[n_{f} \times 1\right], \mathbf{y}_{f}\left[n_{f} \times 1\right], \mathbf{z}_{f}\left[n_{f} \times 1\right]-$ unknown and fixed node coordinates respectively; $\mathbf{z}_{\mathrm{par}}[n \times 1]$ - node coordinates on the offset parabolic surface, $\mathbf{f}_{x}[n \times 1], \mathbf{f}_{y}[n \times 1], \mathbf{f}_{z}[n \times 1]$ - coordinates of the forces acting in the tension ties; $\overline{\mathbf{L}}[r \times r]=\operatorname{diag}(\mathbf{l})-$ matrix of lengths for the first $r$ elements; $\mathbf{T}_{0}[r \times 1]-$ required tension values in the elements. Hereinafter, the horizontal bar above the matrices inidcates that we consider the first $r$ cable elements under the condition of uniform tension.

Connectivity matrix $\mathbf{C}_{\mathrm{s}}\left[m \times n+n_{f}\right]$ for element $e=1 \ldots m$ is:

$$
\mathbf{C}_{s}\left(e, n_{e}\right)=\left[\mathbf{C}, \mathbf{C}_{f}\right]=\left\{\begin{array}{l}
+1, \text { for } n_{e}=i \\
-1, \text { for } n_{e}=j \quad, i<j \\
0, \text { in other cases }
\end{array},\right.
$$

where, $i$ and $j$ are the node numbers of the element $e$.

The solution of the first two equations (1) defines the vectors $\mathbf{x}$ and $\mathbf{y}$ as:

$$
\left\{\begin{array}{l}
\mathbf{x}=\mathbf{D}^{-1}\left(\mathbf{f}_{x}-\mathbf{D}_{f} \mathbf{x}_{f}\right) \\
\mathbf{y}=\mathbf{D}^{-1}\left(\mathbf{f}_{y}-\mathbf{D}_{f} \mathbf{y}_{f}\right) \\
\mathbf{z}(\mathbf{x}, \mathbf{y})=\mathbf{z}_{\mathrm{par}}
\end{array}\right.
$$

where, $\mathbf{D}=\mathbf{C}^{T} \mathbf{Q C}, \mathbf{D}_{f}=\mathbf{C}^{T} \mathbf{Q} \mathbf{C}_{f}$. The third expression in (3) is a vector of node coordinates of the offset parabolic surface (Figure 2). Equation of this surface could be found in [10], [11].

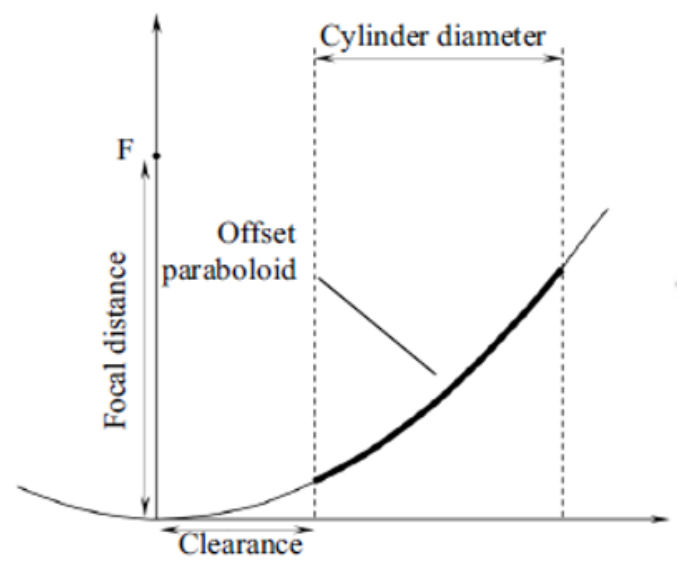

Fig. 3. Offset paraboloid surface diagram

The offset paraboloid parameters values were: cylinder diameter $-12 \mathrm{~m}$; focal distance $5.4 \mathrm{~m}$; clearance $-2.3 \mathrm{~m}$.

The latter equation in (1) specifies the uniform tension restrictions within the first $r$ frontal net cable elements. It is linearized within the initial estimate of force density $\mathbf{q}_{0}$, which leads to the system of indefinite linear equations:

$$
\mathbf{G}^{T} \Delta \mathbf{q}=\mathbf{r}_{0}
$$


where, $\mathbf{r}_{0}=-\left(\overline{\mathbf{L}}\left(\mathbf{x}\left(\mathbf{q}_{0}\right), \mathbf{y}\left(\mathbf{q}_{0}\right), \mathbf{z}\left(\mathbf{q}_{0}\right)\right) \overline{\mathbf{q}}_{0}-\mathbf{T}_{0}\right)$,

$$
\mathbf{G}^{T}=\overline{\mathbf{L}}_{r m}-\overline{\mathbf{Q}} \overline{\mathbf{L}}\left(\overline{\mathbf{U}} \overline{\mathbf{C}} \mathbf{D}^{-1} \mathbf{C}^{T} \mathbf{U}+\overline{\mathbf{V}} \overline{\mathbf{C}} \mathbf{D}^{-1} \mathbf{C}^{T} \mathbf{V}+\overline{\mathbf{W}} \overline{\mathbf{C}} \mathbf{D}^{-1} \mathbf{C}^{T} \mathbf{W}\right) .
$$

Expression (5) is the Jacobian matrix of size $[r \times m]$ which is obtained from the derivative of the function $\overline{\mathbf{L}}(\mathbf{x}(\mathbf{q}), \mathbf{y}(\mathbf{q}), \mathbf{z}(\mathbf{q})) \overline{\mathbf{q}}-\mathbf{T}_{0}$ with respect to $\mathbf{q}$. In-depth description of (5) is in [4]. Matrices $\mathbf{U}[m \times m], \mathbf{V}[m \times m], \mathbf{W}[m \times m]$ were defined as:

$$
\left\{\begin{array}{l}
\mathbf{U}=\operatorname{diag}(\mathbf{u}) ; \\
\mathbf{V}=\operatorname{diag}(\mathbf{v}) ; \\
\mathbf{W}=\operatorname{diag}(\mathbf{w}) ; \\
\mathbf{u}=\mathbf{C x}+\mathbf{C}_{f} \mathbf{x}_{f} ; \\
\mathbf{v}=\mathbf{C y}+\mathbf{C}_{f} \mathbf{y}_{f} ; \\
\mathbf{w}=\mathbf{C} \mathbf{z}+\mathbf{C}_{f} \mathbf{z}_{f} .
\end{array}\right.
$$

Matrix $\overline{\mathbf{L}}_{r m}[r \times m]$ is derived from the matrix $\overline{\mathbf{L}}[r \times r]$ by adding $m-r$ zero columns [9].

The system (4) is indefinite because of $r \leq m$. So, the Lagrangian multiplier method was applied to find the minimum norm solution of $\Delta \mathbf{q}$. This solution was [4]:

$$
\left\{\begin{array}{l}
\lambda_{L}=\left(\mathbf{G}^{\mathrm{T}} \mathbf{G}\right)^{-1} \mathbf{r} ; \\
\Delta \mathbf{q}=\mathbf{G}\left(\mathbf{G G}^{\mathbf{T}}\right)^{-1} \mathbf{r}
\end{array}\right.
$$

where, $\boldsymbol{\lambda}_{L}[r \times 1]-$ is the vector of the Lagrangian multipliers.

Thus, the solution of third equation (1) was determined from the iterative formula:

$$
\mathbf{q}_{k}=\mathbf{q}_{k-1}+\Delta \mathbf{q}
$$

until $\left\|\overline{\mathbf{L}}_{k}-\mathbf{T}_{0}\right\|_{2} \leq \varepsilon$, where, $\varepsilon \leq 0.0001$.

According to (3), the following network shapes were obtained (Figure 4). Nodes at the network vertices were fixed.
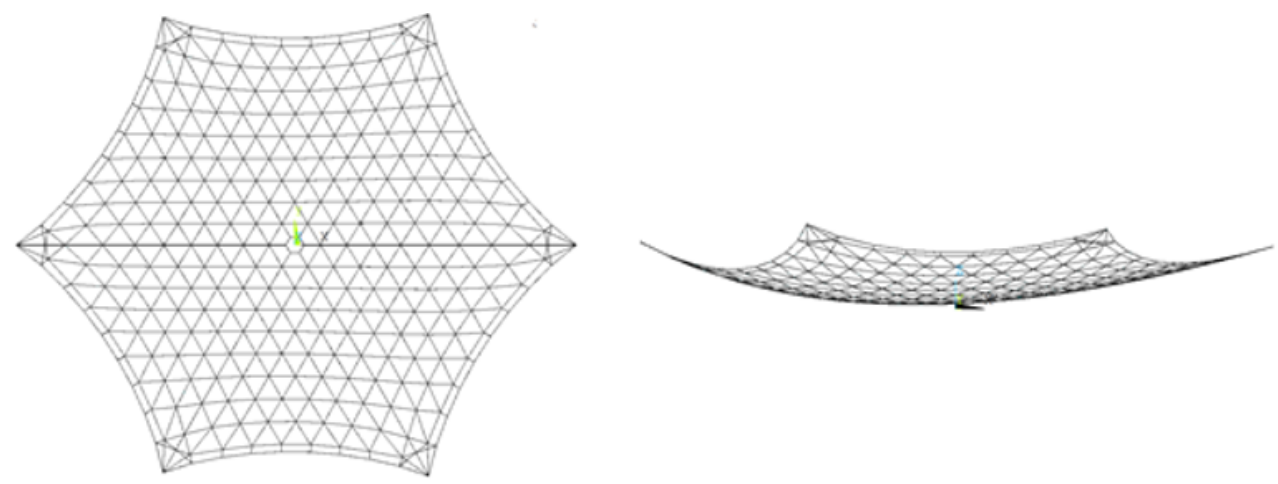

Fig. 4. Calculated reflector frontal (rear) network shapes 
For calculated reflector networks, the matrix $\mathbf{G}^{\mathrm{T}} \mathbf{G}$ was ill-conditioned with the determinant close to zero. This fact was confirmed by the well-known formula of linear algebra:

$$
\operatorname{det}\left(\mathbf{G}^{T} \mathbf{G}\right)=\lambda_{1} \cdots \lambda_{r}
$$

where, $\lambda_{1 \ldots} \lambda_{r}$ - eigenvalues of $\mathbf{G}^{\mathrm{T}} \mathbf{G}$.

Figure 5 illustrates the dependency of $\mathbf{G}^{\mathrm{T}} \mathbf{G}$ eigenvalues to their numbers.

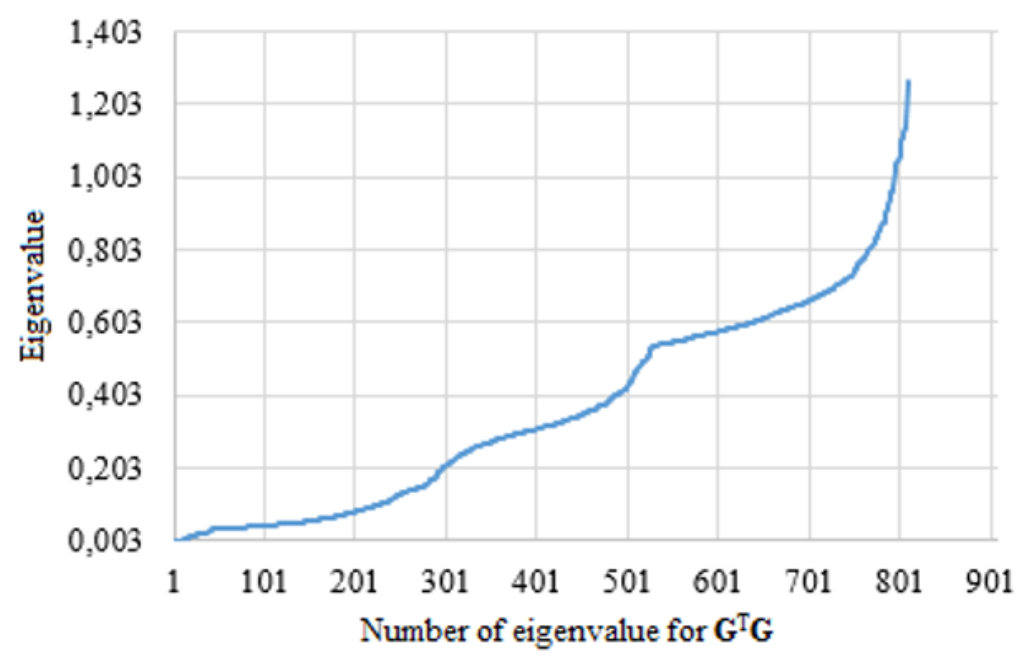

Fig. 5. Dependency of $\mathbf{G}^{\mathrm{T}} \mathbf{G}$ eigenvalues to their numbers.

It is apparent that most eigenvalues are less than $1 . \operatorname{So}, \operatorname{det}\left(\mathbf{G}^{\mathrm{T}} \mathbf{G}\right)$ is close to 0 under matrix dimension $[810 \times 810]$. Thus, in some cases, calculating $\left(\mathbf{G}^{\mathrm{T}} \mathbf{G}\right)^{-1}$ could be a problem. To exclude this problem, Moore-Penrose pseudo-inverse matrix was applied [8]:

$$
\mathbf{A}^{+}=\mathbf{V}_{S V D} \Sigma^{+} \mathbf{U}_{S V D}^{T}
$$

where, $\mathbf{U}_{S V D}\left[m_{\mathrm{p}} \times m_{p}\right], \mathbf{V}_{S V D}\left[n_{p} \times n_{p}\right]-$ orthogonal matrices; $\boldsymbol{\Sigma}^{+}=\operatorname{diag}\left(\frac{1}{\sigma_{1}}, \ldots, \frac{1}{\sigma_{r_{A}}}, 0, \cdots, 0\right)$, $\sigma_{1} \geq \ldots \geq \sigma_{r_{A}} \geq 0$ - singular values of $\mathbf{A}\left[m_{\mathrm{p}} \times n_{p}\right] ; r_{\mathrm{A}}=\operatorname{rank}(\mathbf{A}) ; S V D$ index means singular value decomposition.

The advantage of applying (10) is that it allows to find the minimal norm solution of arbitrary system of linear equations. If, for square matrix $\mathbf{A}, \operatorname{det}(\mathbf{A}) \neq 0$, then [8]:

$$
\mathrm{A}^{+}=\mathrm{A}^{-1}
$$

So, matrix $\mathrm{A}+$ is the generalization for inverse matrix. It means that we can use the following relations for finding $\Delta \mathbf{q}$ :

$$
\left\{\begin{array}{l}
\lambda_{L}=\left(\mathbf{G}^{\mathbf{T}} \mathbf{G}\right)^{+} \mathbf{r} \\
\Delta \mathbf{q}=\mathbf{G}\left(\mathbf{G G}^{\mathbf{T}}\right)^{+} \mathbf{r}
\end{array}\right.
$$




\section{Stage 2: Calculation of reflector node displacements}

\subsection{Verification of reflector frontal (rear) network shapes calculated by non- linear FDM}

Figures 6, 7 illustrate the reflector frontal (rear) network node displacements and calculated tension values in the FEM of cables.

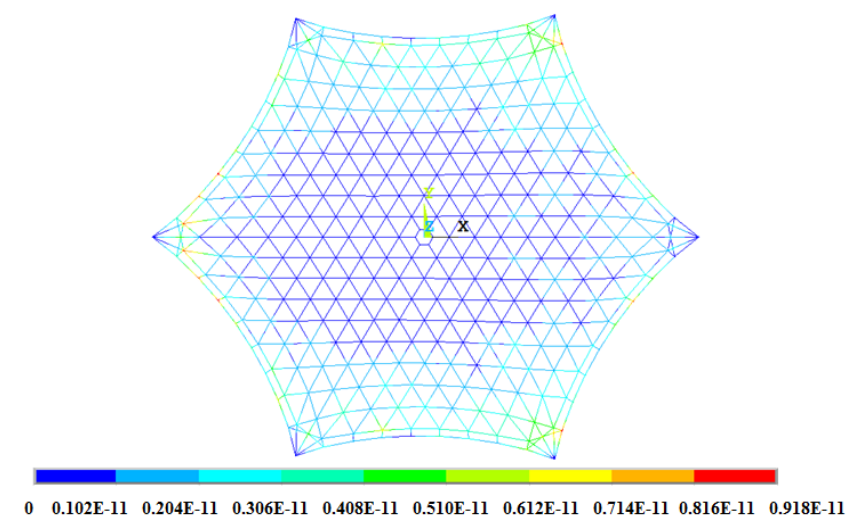

Fig. 6. Node displacements in the FEM of frontal (rear) cables, $m$
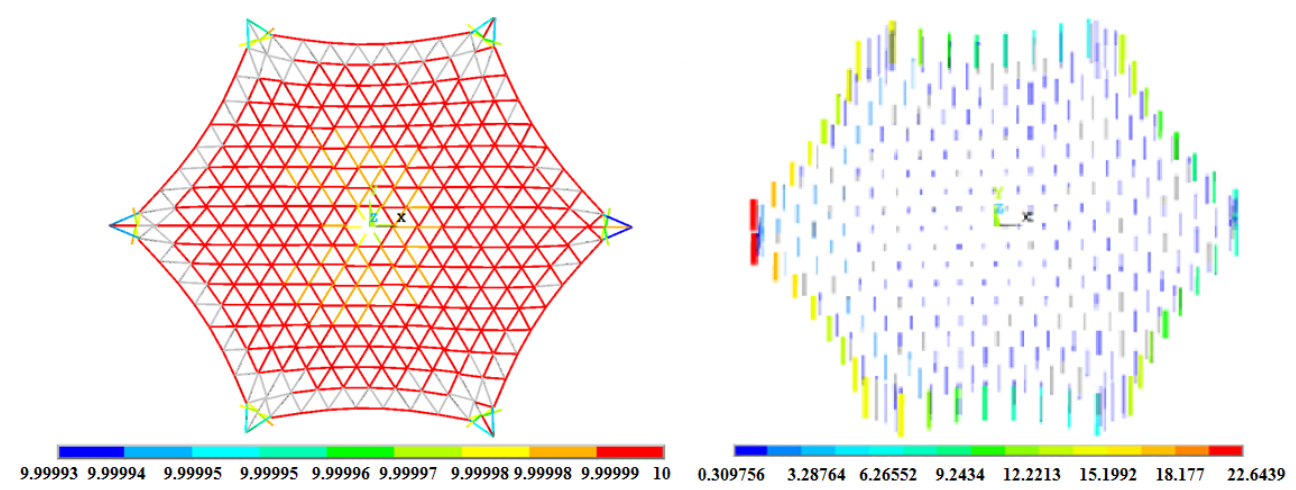

Fig. 7. Tension distribution in the FEM of frontal (rear) nets and tension ties, $\mathrm{N}$

It is apparent that node displacements are practically zero, while the tension values of frontal (rear) cable elements are close to required value $10 \mathrm{~N}$. So, the obtained solution of equations (1) is correct.

\subsection{Calculation of reflector node displacements}

Based on found reflector frontal (rear) net and corresponding boundary conditions (values of cable and mesh initial tension as well as node zero displacement boundary conditions at the framework hub), the mesh node displacements fields were obtained (figure 8): 


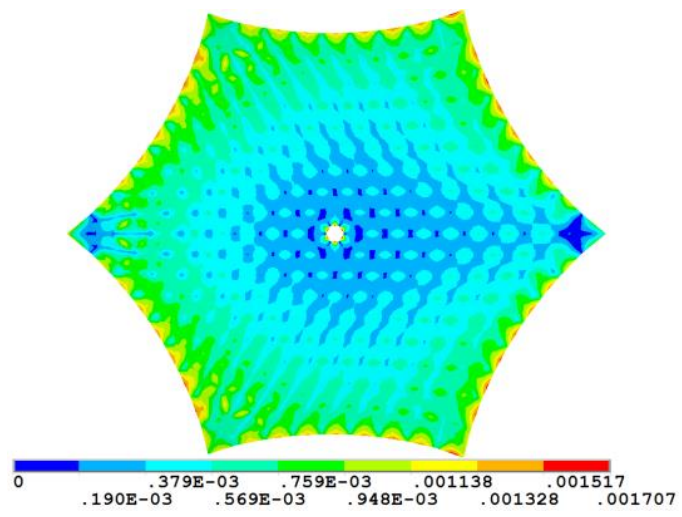

Fig. 8. Mesh node displacement fields, $m$

The reflector FEM characteristics were the following: mesh thickness $-0.1 \mathrm{~mm}$; cable diameters - no more than $2 \mathrm{~mm}$; the order of magnitude of elasticity modules: mesh $10^{6} \mathrm{~Pa}$, cables $-10^{9} \mathrm{~Pa}$; framework spokes $-10^{11} \mathrm{~Pa}$.

Figure 9 shows a distribution of $\Delta z_{j}^{\mathrm{par}}=z_{j}-z_{j}^{\mathrm{par}}$ values:

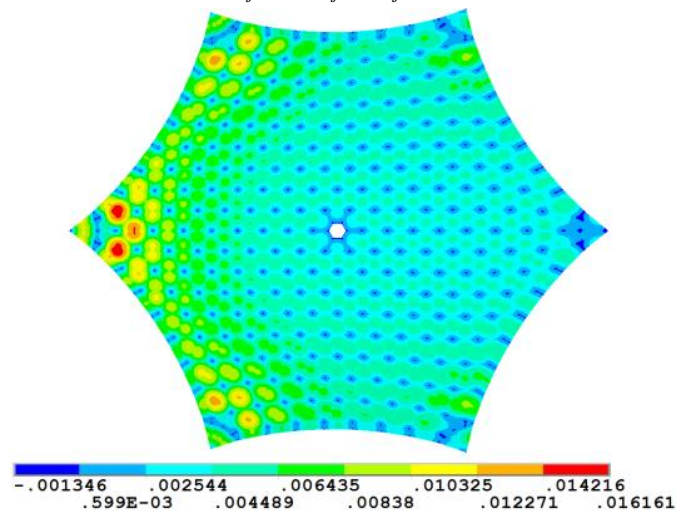

Fig. 9. Distribution of $\Delta z_{j}^{\text {par }}$ values in the reflective mesh, m

where, $z_{j}=z_{j}^{F E M}+u_{j}^{z}, z_{j}^{F E M}, u_{j}^{z}$ - node coordinate of FEM and mesh node displacement on $\quad$ z-axis respectievly; $\quad z_{j}^{\mathrm{par}}=z_{j}^{\mathrm{par}}\left(x_{j}^{F E M}+u_{j}^{x}, y_{j}^{F E M}+u_{j}^{y}\right)-$ node coordinate on the theoretical offset parabolic surface; $j=1 \ldots N$ - node number.

Root-mean square (RMS) error value of mesh nodes from the offset parabolic surface was calculated as:

$$
\delta_{R M S}=\left[\frac{1}{N} \sum_{j=1}^{N}\left(\Delta z_{j}^{R M S}\right)^{2}\right]^{1 / 2}
$$

where, $\Delta z_{j}^{R M S}=z_{j}-\left(z_{j}^{\mathrm{par}}+M\right), M=\sum_{j=1}^{N}\left(z_{j}-z_{j}^{\mathrm{par}}\right) / N-$ average node deviation from the the theoretical offset parabolic surface on z-axis. The value (13) is an essential parameter, i.e. it has direct relevance to the reflector gain [10]. For the considered reflector structure it was $2.5 \mathrm{~mm}$, but in the circle of radius $6.1 \mathrm{~m}$ it was $2 \mathrm{~mm}$. 
The RMS error could be calculated in different ways, for example, using the best fit parabolic surface [11].

It should be noted that applying FDM solution made it possible to determine the reflector node displacement field with imposed boundary conditions. However, there could be cases of convergence problems in FEM under imposed boundary conditions. In this case, the procedure of sequence node displacement boundary condition changes could be applied [3].

\section{Conclusion}

The paper describes the numerical modeling of space umbrella-type mesh reflector. The first stage included the calculation of cable node element coordinates by the non-linear FDM. Based on the eigenvalue spectrum, it was determined that $\mathbf{G}^{\mathrm{T}} \mathbf{G}$ matrix was illconditioned for the network in figure 4 . Thus, to exclude the possible convergence problem for the iterative formula (8), the Moore-Penrose pseudo-inverse matrix was applied. The FDM solution results were verified by the appropriate FEM solutions for the cable nets (figures 6, 7).

In the second stage of the modeling, found reflector frontal (rear) net shapes and corresponding values of cable tension were used as an initial estimate in determining reflector node displacement field (figure 8). It was found that the RMS error value (13) for the mesh nodes was $2.5 \mathrm{~mm}$ for the whole mesh area and $2 \mathrm{~mm}$ in the circle of radius $6.1 \mathrm{~m}$.

In the cases of convergence problems for FEM under imposed node boundary conditions, the procedure of sequence node displacement boundary condition changes [3] could be used.

This work has been supported by Ministry of Science and Higher Education of the Russian Federation. Agreement No 14.578.21.0257. Unique identifier RFMEFI57817X0257.

\section{References}

1. W.A. Imbrial, S. (Shichang) Gao, L. Boccia, Space Antenna Handbook (John Wiley \& Sons, Ltd, Chichester, 2012)

2. N.H. Kim, Introduction to Nonlinear Finite Element Analysis (Springer, New -York Heidelberg Dordrecht London, 2015)

3. S. Belov, M. Pavlov,V. Ponomarev, S. Ponomarev and A. Zhukov, AIP Conf. Proc. 1772, 010001 (2016); doi: 10.1063/1.4964522 (2016)

4. H. Schek, Comput. Method. Appl. Mech. Eng. 3, 115-134 (1974)

5. S. Adriaenssens, P. Block, D. Veenendaal, and C. Williams, Shell structures for architecture: form finding and optimization (Routledge, Oxon, 2014)

6. H. Tanaka, M.C. Natori, J., Acta Astronaut. 55, 154-163 (2004)

7. M. Quagliaroli and P. G. Malerba, Int. J. Solids Struct. 50, 2340-2352 (2013)

8. G.H. Golub, C.F. Van Loan, Matrix Computations (The Johns Hopkins University Press, 2013)

9. G. Tibert, Numerical analyses of cable roof structures (Lic. Thesis, Royal Institute of Technology, Stockholm, 1999)

10. G. Tibert, Deployable tensegrity structures for space applications, (Doctoral Thesis, Royal Institute of Technology, Stockholm, 2002)

11. S. Morterolle, B. Maurin, J. Quirant and C. Dupuy, Acta Astronaut. 76, 519-527 (2012) 